\title{
A Conceptual Framework for Designing Smart Domestic Health Enhancement Devices
}

\author{
Pei Fen CHEE, Jo-Yu KUO ${ }^{1}$ and Chun-Hsien CHEN \\ School of Mechanical and Aerospace Engineering, Nanyang Technological University, \\ Singapore
}

\begin{abstract}
The process of product design and development can be complex, especially in transdisciplinary practice. A design framework is often adopted to better govern this process. In recent years, there is a growing trend for domestic devices promoting health enhancement. However, due to its specific functionality and a strong need for safety emphasis, domestic health enhancement devices need to be distinguished from other domestic consumer products from the initial stages of designing. Therefore, this study aims to explore the influence of design principles on the artefacts by developing a framework for the design and development of domestic health enhancement devices. It adopts the approach of targeting a selected lifestyle or demography and tailoring a set of device functionalities to its needs. Throughout the process, the proposed framework provides disciplined guidelines to achieve its three design objectives - safety, effectiveness, and user receptiveness. A case study is performed to demonstrate the application of the proposed framework in the context of high heel wearers. The sensor-based real-time feedback is integrated into the user involved design process. The first design iteration in accordance to the framework is completed and evaluated.
\end{abstract}

Keywords. Design framework, domestic health enhancement device, product design and development, transdisciplinary

\section{Introduction}

Technological advances have encouraged a stay-home lifestyle. Indoor entertainment such as video streaming and online shopping removes the physical need to leave the comfort of home. The result is a great potential to escalate into a sedentary lifestyle, which is closely associated with increasing chronic and lifestyle diseases. Hence, there is a growing trend for domestic devices promoting health enhancement in recent years. This is partly attributed to an increasing health awareness along with widely available health information. The emergence of the COVID-19 pandemic further supplemented this appetite as people are spending more time at home, where become the all-in-one place for work, play, exercise, etc.

However, the design and development of domestic health enhancement devices may encounter various setbacks due to its specific nature. This category of devices needs to be distinguished from that of other domestic consumer products, with a specific set of functionality and a much stronger emphasis on safety [1].

\footnotetext{
${ }^{1}$ Corresponding Author, Mail: jykuo@ntu.edu.sg
} 


\section{Literature Review}

\subsection{Domestic health enhancement devices}

A domestic health enhancement device is to be differentiated from a "home medical device". They are similar in terms of "use in a nonclinical or transitory environment, which is managed partly or wholly by the user, requires adequate labelling for the user, and may require training for the user by a health care professional in order to be used safely and effectively" [2]. While the latter is intended "for use in the diagnosis of disease or other conditions, or in the cure, mitigation, treatment or prevention of disease", domestic health enhancement, for the scope of this project, focuses solely on independent illness prevention.

A huge range of domestic health enhancement devices exists in the market. This is due to different approaches catering to different market segments. Firstly, general health enhancement devices are of the widest variety in the market. They range from large equipment such as treadmills to small portable dumbbells and yoga blocks. However, uninformed use of such devices may cause aggravation or discomfort to these users with pre-existing health conditions.

Secondly, condition-specific health enhancement devices are of medical nature, ranging from prevention and treatment to rehabilitation. Depending on the severity of the condition, professional supervision may be necessary to ensure patient safety [3]. Clinical directions and supervision are necessary to ensure patient safety, making stretching unsuitable for domestic implementation. Without deep interest and profound expertise, it is difficult to achieve under domestic conditions.

Lastly, the demography/lifestyle-specific devices serve a defined target group, along with its associated symptoms and risk hazards. These symptoms tend to be mild in nature, as they have yet to develop into more severe health conditions. Therefore, solutions are largely preventive in nature, and are safer to administer in the domestic context. Furthermore, a domestic solution is ideal for this market segment, as people tend to not seek clinical aid for mild discomfort.

\subsection{Design frameworks}

Designers nowadays encounter transdisciplinary challenges more than ever. Design frameworks can be useful as a guiding strategy providing a clear structure to allow them to communicate with stakeholders in a more holistic way [4]. It supports a broader take on new product development, where every decision in the product life cycle has an impact on innovation [5]. Pragmatic considerations, including but not limited to technical, economic and company strategic decisions, highlight the importance of an efficient and competitive design process. Hence, these frameworks tend to suggest applying an iterative procedure to review internal and external sources of knowledge.

Some design frameworks are largely user-centric [6], for specific demography such as the older workforce [7], or promoting a pleasurable interaction between human and technology [8]. The latter asserts that upon the satisfaction of ergonomic factors - safety and functionality, designers should account for hedonomic factors - usability, pleasurable experience and individuation. This is interpreted as a concise hierarchy of 
needs in design and development, with an emphasis on the appeal of customizability offering a value adding edge to user appeal.

Individual motivation is cited as an important factor to engagement and eventually recovery for a home-based rehabilitation system [9]. Methods to achieving motivation include inspiration, manageability, benefit awareness and progress awareness through feedback. Notably, sensor feedback is also helpful in detecting unsafe compensatory movements, thereby ensuring the quality of engagement [10].

Understanding the characteristics of various existing product design frameworks is essential in assessing their applicability for domestic health enhancement devices. A tailored framework is recommended to guide safe and effective designs due to its many unique features. Furthermore, the influence of the design frameworks on the artefacts has not been fully understood. This paper is interested in crafting a framework to incorporate the specific design principles to facilitate the design and development stage.

\section{Method}

This project aims to outline a conceptual framework for the ease of use by designers of domestic health enhancement devices. The proposed framework is depicted in Figure 1.

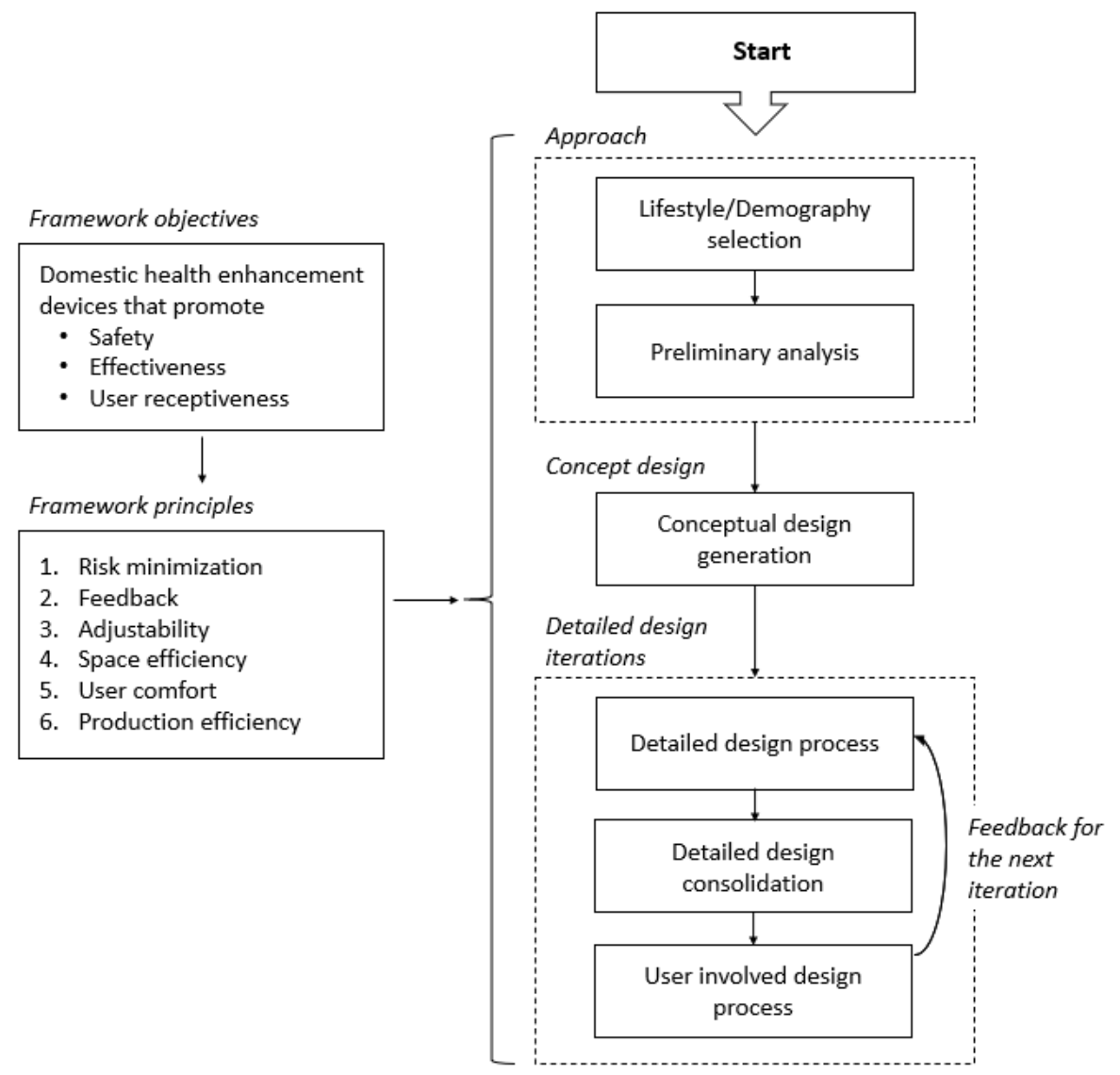

Figure 1. Overview of the conceptual framework. 
The left side of the framework details the predetermined objectives and principles, which compile the collective concerns of designing for domestic health enhancement. They serve to govern the entire design and development process.

The right side of the framework outlines the path that future designers can follow in their design process. The process of conceptual design generation depends on the preference of each product designer. Common tools such as functional analysis, morphological charts, decision matrix can be useful.

An advice integrated in the framework is to begin the design process targeting a lifestyle or demography. This eliminates the outcome possibilities of generic products that are prone to misuse, or condition-specific devices that are unsuited for domestic implementation. Preliminary analysis should be complete and thorough with the following:

- Short- and long-term health implications of lifestyle or demographic group.

- Expert and clinical recommendations, and if an unsupervised activity is safe.

- Analysis of existing solutions evaluated with framework principles.

\subsection{Identify framework principles}

There are three objectives identified for the scope of domestic health enhancement devices. Safety is the top priority of the framework. The goal of domestic health enhancement means that device engagement will occur without professional supervision. Extra precautions must be made throughout the design and development stage to ensure minimal risk for the users. Effectiveness is a measure of degree that the device can achieve its purported functionality, given that the device is used ideally as intended. User receptiveness can be defined as the level of inclination that users will engage with the device. It is affected by various hedonomic factors, including user experience and personalization option. Six design principles are then derived. Figure 2 is a pictorial representation of the relationships between objectives and principles.

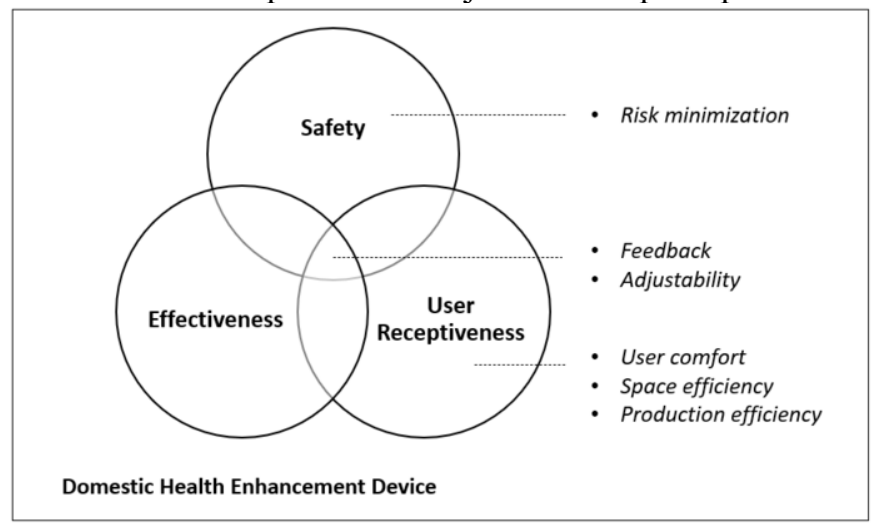

Figure 2. Relationship between design objectives and principles.

\subsection{Detailed design iterations}

Notably, the detailed design stage involves multiple iterations to optimize device design, where user feedback is continually injected for improvement. Each iteration is complete with a rigorous detailed design process (Figure 3), detailed design consolidation and 
feedback from user involvement. The last stage of the detailed design process should clearly outline areas requiring user input and assess the level of maturity, with design principles largely satisfied and details complete with production considerations.

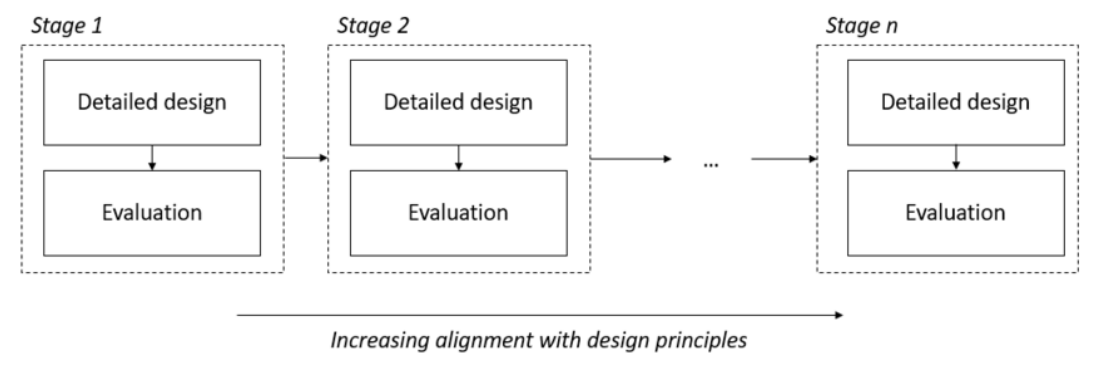

Figure 3. Detailed design iteration process.

In the interest of time and efficiency, the idea should proceed to the next stage once both criteria are met. Detailed design consolidation includes engineering concerns such as material selection and dimensioning, and user interaction considerations in the form of a user manual. While the improvement process can be endless through prototype and experimentation, the device can be considered market ready once all framework objectives are met i.e., safety, effectiveness, and user receptiveness.

\section{Case study}

A case study on the design and development of domestic health enhancement devices for High Heeled Shoe (HHS) wearers is performed to demonstrate the use of the proposed framework. HHS wearers encounter both temporary discomfort and long-term health implications. The severity of these symptoms varies according to the characteristics of HHS selected, in terms of heel height and width of heel base and tip. Hence, they make an ideal group to introduce domestic options that promote both pain relief and health enhancement.

\subsection{Preliminary Analysis}

Firstly, the main causes of HHS health implications are identified. The posture and gait are altered during normal walking, generating cause muscle fatigue in the calf gastrocnemius muscles [11]. Elevated heel height also puts additional pressure on the wearers' knees due to a larger knee joint extensor moment [12]. This leads to a large increase in bone-on-bone forces in the knee joint and may increase the risk of degenerative joint and musculoskeletal disease [13]. Functional mobility including movement velocity, directional control and excursions are compromised at a heel height of $7 \mathrm{~cm}$, which standing balance worsens at $10 \mathrm{~cm}$ [14].

The fact that HHS wearers tend to shift the body's centre of mass forward during both standing and walking concentrates pressure at their forefoot. This may result in the development of metatarsalgia [15]. Moreover, long-term HHS wearing can cause stiffening of the Achilles tendon and shortening in the medial gastrocnemius muscle fascicles [16]. This adaption has several implications - a smaller ankle's active range of 
motion and greater angles of ankle plantarflexion at rest, as well as reduced force generation capacity [17].

As a consequence, expert and clinical recommendations are made to alleviate the implications such as to avoid aggravation in spinal disks and knee joints associated with high impact activities, stretch calf to prevent calf shortening and aid in pain relief, and stretch ankle to increase eversion range of motion for stability [18]. Products marketed to address symptoms include but are not limited to adjustable stretch boards, resistant bands, and metatarsal pad insoles.

\subsection{Concept design}

To analyse the intended functions of the device, the functional analysis is employed and two main functions are shortlisted.

- Dorsiflexion stretching, which offers both short term pain relief in foot and calf tightness, as well as lasting benefits against calf shortening (Framework design principle: Effectiveness, User comfort)

- Eversion stretching, which works on the same leg region (Framework design principle: Space efficiency)

Next, sub functions are identified to set the direction of design thinking.

- Adjustable device to accommodate different anthropometrics and flexibility (Framework design principle: Adjustability)

- $\quad$ Provide feedback to enable motivation, track progress and integrate safety limits (Framework design principle: Feedback, Risk minimisation)

- Integrate soft padding to prevent aggravation in stressed areas (Framework design principle: Risk minimisation, user comfort)

A morphological chart is derived based on the key technical function identified in functional analysis. Table 1 shows the possible solutions for adjusting device dimensions.

Table 1. Possible solutions in the morphological chart.

\begin{tabular}{llll}
\hline \multicolumn{1}{c}{ Solution 1} & \multicolumn{1}{c}{ Solution 2 } & Solution 3 & Solution 4 \\
\hline $\begin{array}{l}\text { Adjustable angle by } \\
\text { adjusting rod's position } \\
\text { in groove }\end{array}$ & $\begin{array}{l}\text { Adjustable length using } \\
\text { telescopic tubes, which } \\
\text { are secured by a bolt }\end{array}$ & $\begin{array}{l}\text { Rounded frame that } \\
\text { can be adjusted readily } \\
\text { to reach a new } \\
\text { equilibrium }\end{array}$ & $\begin{array}{l}\text { Frame adjustment } \\
\text { through moving pivots } \\
\text { to reach new } \\
\text { equilibrium }\end{array}$ \\
\hline
\end{tabular}

Three designs are conceptualized based on the morphological chart. A rating value is accorded to each concept based on how well it meets the criteria. Rating values range from a scale of 1 to 5,1 being very poor and 5 being ideal. Rating values are subsequently multiplied by the weights to generate weighted values (W.V.). Using a decision matrix approach, one final conceptual design is shortlisted (Table 2), in accordance with its performance in the framework design principles. 
Table 2. Concept evalutaion based on the decision matrix.

\begin{tabular}{lcccccc}
\hline \multirow{2}{*}{ Criteria } & W.V. & Value & W.V. & Value & W.V. \\
\cline { 2 - 6 } & Value & 90 & 4 & 120 & 5 & 150 \\
\hline High safety (30\%) & 3 & 40 & 3 & 60 & 5 & 100 \\
\hline High effectiveness (20\%) & 2 & 20 & 5 & 50 & 5 & 50 \\
\hline High comfort level (10\%) & 2 & 20 & 4 & 80 & 2 & 40 \\
\hline High ease of use (20\%) & 1 & 50 & 4 & 40 & 1 & 10 \\
\hline Low Cost (10\%) & 5 & 30 & 4 & 40 & 1 & 10 \\
\hline Min maintenance (10\%) & 3 & 250 & & 390 & 360 \\
\hline Sum of weighted values & & 50 & & 5 \\
\hline
\end{tabular}

\subsection{Detailed design process - iteration 1}

In this case study, iteration 1 is defined in three stages (Figure 4). Stage 1 design caters to the user being in the most comfortable position without having to shift in between modes of use. During dorsiflexion, the user leg is extended for maximum effectiveness. During eversion, the user bends her knees and have a firm footing. In Stage 2, changes of pivot plot are made accordingly to compact the mechanism. Platforms are redesigned to accommodate ankle during dorsiflexion. Cushion height is also accounted for to reserve space for ankle. To reduce the size of the device while maintaining the advantage that user does not have to shift positions, a dynamic design is proposed in Stage 3.

The most significant change from Stage 1 to 3 is in its size, an important consideration for domestic devices (Framework design principle: Space efficiency). Padding and flexibility in device have also been improved (Framework design principle: User comfort, Adjustability).
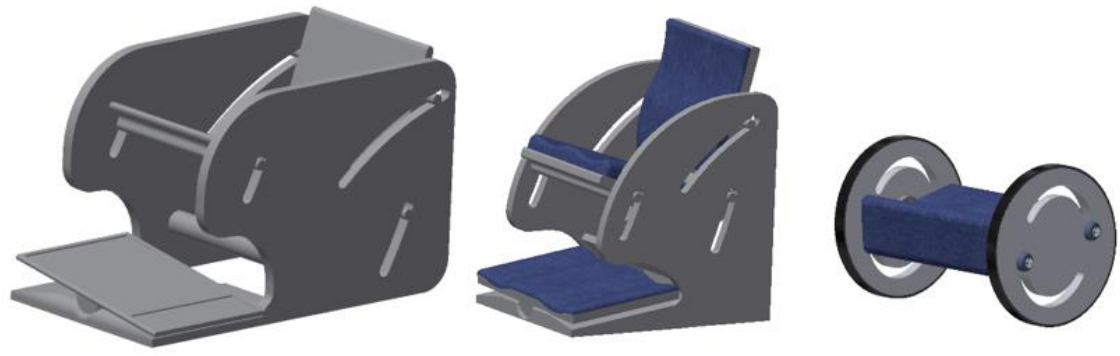

Figure 4. Iterative improvements from Stage 1 (left) to Stage 3 (right).

The intended setting of use is from a seated position (Figure 5). A simplified static structural analysis was performed on the state of the device when a user (seated at $450 \mathrm{~mm}$ seat height) rests her legs on the foot platform, with zero dorsiflexion. Since strain in the device is minimal and negligible, there will be no plastic deformation nor changes to device operations. In summary, key features of the design include: 
- Diversion of weight of leg to dorsiflex foot.

- Moving pivots in the dynamic frame to accommodate a range of flexibility and anthropometrics.

- Lever mechanism to facilitate ankle eversion stretching.

- Sensors delivering real-time feedback through tilt angles and applied force.
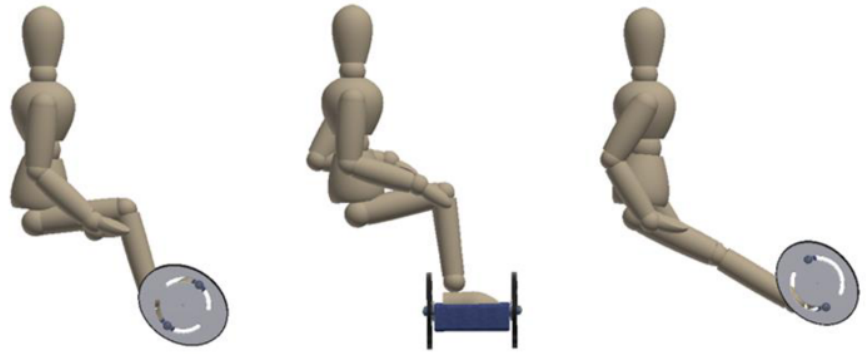

Figure 5. The flexed knee dorsiflexion (left), eversion (centre), extended leg dorsiflexion (right).

In accordance to the framework, the design is ready for user testing since it has largely improved and satisfied design principles. Concise areas that require user inputs include stretching effectiveness quantified by dorsiflexion angles, and subjective assessment of user comfort level with the novel dynamic frame.

Prototype making furthered manufacture considerations and design maturity. The experiment setting simulates the intended engagement setting (Figure 6). Two types of sensors were used concurrently to track the user's dorsiflexion activity. Tilt sensors were employed to detect the incline angle when the foot platform was engaged. To ensure that the tilt detected was associated with user activity, force sensors were placed behind each ankle to detect pressure from the ankle when the foot platform was pushed down.

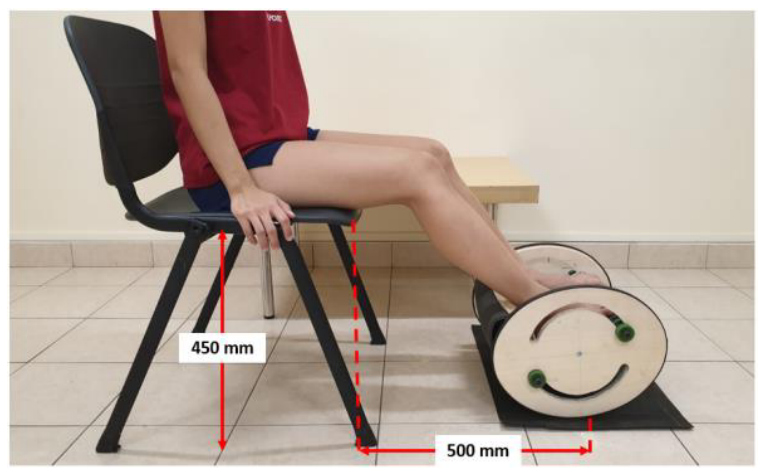

Figure 6. Experiment setting.

The participants were asked to exercise in a sequence. They began with the resting position, dorsiflexion mode (flexed knee) with a manual counter of 1 minute, eversion mode, dorsiflexion mode (extended leg) with a sensor-activated counter of 1 minute, and then return to resting position. Along the process, real-time feedback is accorded to different levels of user engagement - "Not in use", "Push ankle down" during the insufficient stretch, counting when sensor threshold is met, as well as "Very good" with counting when exceeding sensor threshold.

In total, 44 dorsiflexion angles were tracked in the experiment, with 22 female participants (age between 19 to 25 years old) and both feet taken as separate data. The 
results showed a normal distribution of change in dorsiflexion angle (Figure 7). It reveals a positive mean value of 1.7, suggesting the stretching effectiveness of the device in a singular use. A relatively large standard deviation at 3.0 may be attributed to individual factors such as lack of motivation or uneven stretch in left and right foot due to existing different flexibilities (e.g. ankle sprain history).

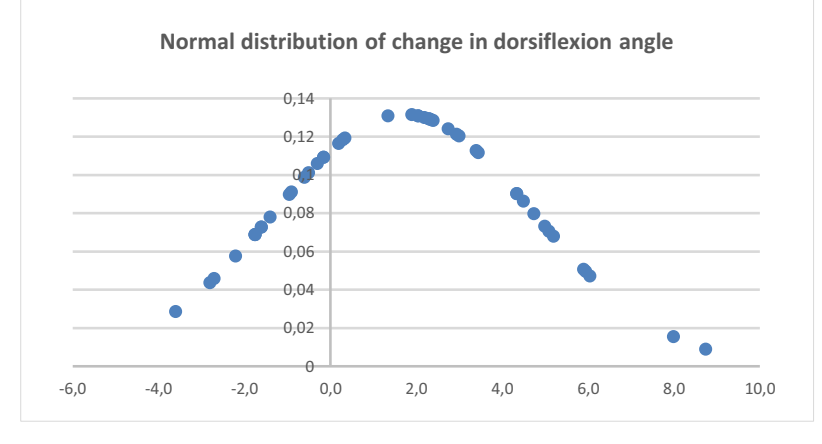

Figure 7. Normal distribution of change in dorsiflexion angle.

Additionally, over $75 \%$ of participants have rated at least four out of five for the usefulness of feedback in motivating dorsiflexion activity. This emphasises the importance of feedback as a framework design principle. Both objective and subjective results from the experiment set the direction for the next iteration of detailed design. Room for improvement, along with suggestions, is listed below.

- Presence of stretch beyond the intended region necessitates a professional reevaluation of safety limits.

- Reconsideration of device centre to reduce additional user effort required to stabilise the dynamic frame.

- Improve the frame shape to minimise the biased eversion stretching due to device asymmetry.

- Comprehensive visual or physical indicators (e.g. arrows and handles) to facilitate an intuitive use of the novel dynamic framework.

\section{Conclusion}

This study explores a novel approach to develop a conceptual framework that facilitates the attainment of three design objectives - safety, effectiveness, and user receptiveness. The proposed framework puts an emphasis on increasing alignments with design principles during the design iteration process. This is done by identifying and analysing the gaps in existing frameworks in addressing the design and development of domestic health enhancement devices.

The proposed framework proves to be helpful in the case study from topic selection, concept generation to detailed design and user-involved experiments. A case study has demonstrated the use of the framework in guiding the early stages of design up to recommendations for the second design iteration. The results are channelled to begin the next iterative process to improve the device design.

Continual professional consultations with healthcare practitioners should be included to enhance the reliability of the framework design process. There is also an 
unaddressed contradiction between user receptiveness and effectiveness, which is likely to surface in the later iterations of designs. Further studies are needed to bring it to completion for a systematic hierarchy in design objectives and greater generalizability.

\section{Acknowledgement}

This paper is supported by School of Mechanical \& Aerospace Engineering, Nanyang Technological University, under the project Human Factors, Ambient Intelligence and Ambient Assisted Living for Aging Population in Asia (Project ID: \#001254-00001).

\section{References}

[1] N. R. Council, The role of human factors in home health care: Workshop summary. Nat. Acad., 2010.

[2] N. Ear and T. D. Branch, Guidance for Industry and FDA Staff Regulatory Requirements for Hearing Aid Devices and Personal Sound Amplification Products, U.S. Department of Health and Human Services Food and Drug Administration Center for Devices and Radiological Health, 2009.

[3] H. Alfredson, T. Pietilä, P. Jonsson, R. Lorentzon, Heavy-load eccentric calf muscle training for the treatment of chronic Achilles tendinosis, The Am. journal of sports medicine, 1998, 26(3), pp. 360-366.

[4] W. Song and T. Sakao, A customization-oriented framework for design of sustainable product/service system, Journal of Cleaner Production, 2017, Vol. 140, pp. 1672-1685.

[5] C. Vila and J. C. Albiñana, An approach to conceptual and embodiment design within a new product development lifecycle framework, Int. Journal of Production Research, 2016, 54 (10), pp. 2856-2874.

[6] X. Sun, R. Houssin, J. Renaud, and M. Gardoni, A review of methodologies for integrating human factors and ergonomics in engineering design, International Journal of Production Research, 2019, Vol. 57, No. 15-16, pp. 4961-4976.

[7] I. Gonzalez and P. Morer, Ergonomics for the inclusion of older workers in the knowledge workforce and a guidance tool for designers, Applied Ergonomics, 2016, Vol. 53, pp. 131-142.

[8] P. A. Hancock, A. A. Pepe, and L. L. Murphy, Hedonomics: The power of positive and pleasurable ergonomics, Ergonomics in design, vol. 13, no. 1, pp. 8-14, 2005.

[9] S. Rennick Egglestone, L. Exelrod, T. Nind, R. Turk, A. Wilkinson et al., A design framework for a home-based stroke rehabilitation system: Identifying the key components, 2009 3rd International Conference on Pervasive Computing Technologies for Healthcare: IEEE, 2009, pp. 1-8.

[10] Y. Wang, S. Yu, and N. Ma, A requirement-scenario-experience framework for evaluating wearable and fashionable design: Presenting underlying factors of user loss, International Journal of Industrial Ergonomics, 2018, Vol. 68, pp. 137-148.

[11] J. Yu, D. W.-C. Wong, H. Zhang, Z.-P. Luo, and M. Zhang, The influence of high-heeled shoes on strain and tension force of the anterior talofibular ligament and plantar fascia during balanced standing and walking, Medical engineering \& physics, 2016, Vol. 38, No. 10, pp. 1152-1156.

[12] K. Y. Chew, Y. Q. Tan, S. C. Chan, The effects of different high heeled shoes during gait at the kinetic and kinematic impact, 2017 IEEE 15th Student Conf. on Research and Development, 2017, pp. 481-486.

[13] E. B. Simonsen, M.B. Svendsen et al., Walking on high heels changes muscle activity and the dynamics of human walking significantly, Journal of applied biomechanics, 2012, Vol. 28, No. 1, pp. 20-28.

[14] V. D. Hapsari, S. Xiong, and S. Yang, High heels on human stability and plantar pressure distribution: Effects of heel height and shoe wearing experience, Proceedings of the Human Factors and Ergonomics Society Annual Meeting, 2014, Vol. 58, No. 1, pp. 1653-1657.

[15] D. Y. Lee, D.-O. Lee, and H.-G. Jung, Metatarsalgia and toe deformities, in H.-G. Jung (ed.) Foot and Ankle Disorders, Springer, Berlin Heidelberg, 2016, pp. 77-119.

[16] R. Csapo, C. Maganaris, O. Seynnes, and M. Narici, On muscle, tendon and high heels, Journal of Experimental Biology, 2010, Vol. 213, No. 15, pp. 2582-2588.

[17] A. Farrag and W. Elsayed, Habitual use of high-heeled shoes affects isokinetic soleus strength more than gastrocnemius in healthy young females, Foot \& ankle international, 2016, 37(9), pp. 1008-1016.

[18] Y. Kim, J.-M. Lim, and B. Yoon, Changes in ankle range of motion and muscle strength in habitual wearers of high-heeled shoes, Foot \& ankle international, 2013, Vol. 34, No. 3, pp. 414-419. 